\title{
Influência da paridade no desenvolvimento da depressão em mulheres brasileiras
}

\author{
Influence of parity on the development of depression in Brazilian women \\ Influencia de la paridad en el desarrollo de la depresión en mujeres brasileñas
}

Recebido: 31/03/2021 | Revisado: 10/04/2021 | Aceito: 13/04/2021 | Publicado: 24/04/2021

\author{
Adriana Gomes Magalhães \\ ORCID: https://orcid.org/0000-0002-0279-5930 \\ Universidade Federal do Rio Grande do Norte, Brasil \\ E-mail: adriana_fsm@yahoo.com.br \\ Damião Ernane de Souza \\ ORCID: https://orcid.org/0000-0002-2038-9397 \\ Instituto Brasileiro de Geografia e Estatística, Brasil \\ E-mail: souzade73@gmail.com \\ Wanessa Cristina Tomaz dos Santos Barros \\ ORCID: https://orcid.org/0000-0002-1924-3278 \\ Universidade Federal do Rio Grande do Norte, Brasil \\ E-mail: wanessaenf@bol.com.br \\ Laiane Santos Eufrásio \\ ORCID: https://orcid.org/0000-0003-0578-7140 \\ Universidade Federal do Rio Grande do Norte, Brasil \\ E-mail: 1aieufrasio@hotmail.com \\ Leonildo Santos do Nascimento Júnior \\ ORCID: https://orcid.org/0000-0002-5383-8494 \\ Universidade Federal do Rio Grande do Norte, Brasil \\ E-mail: leonildofisio@gmail.com \\ Elizabel de Souza Ramalho Viana \\ ORCID: https://orcid.org/0000-0002-2692-9251 \\ Universidade Federal do Rio Grande do Norte, Brasil \\ E-mail: elizabelviana@gmail.com
}

\begin{abstract}
Resumo
Objetivo: Analisar a relação entre paridade e depressão em mulheres em idade reprodutiva entrevistadas na Pesquisa Nacional de Saúde, 2013. Metodologia: Estudo transversal, de base populacional realizado com base nos dados da Pesquisa Nacional de Saúde Brasileira (PNS) - 2013. Foram incluídos registros de mulheres em período reprodutivo, com idade entre 18 e 49 anos, totalizando a amostra com 15.344 mulheres. Resultados: Observou-se associação bruta estatisticamente significante entre a paridade e depressão ( $\mathrm{RP}=1.32$; IC 95\%: 1.20 - 1.46). Houve também associação entre ter filhos com baixo peso e depressão ( $\mathrm{RP}=1.64$; IC 95\%: 1.24 - 2.18). Mostrando que mulheres em idade reprodutiva mais velhas e com 3 filhos ou mais apresentam 36 vezes mais chances de referir diagnóstico médico de depressão, e aquelas que tiveram filho com baixo peso essa probalidade quase se duplicou. Conclusão: Mulheres em idade reprodutiva mais avançada, que tiveram maior paridade, tiveram mais chances de apresentarem depressão, havendo uma potencialização dessa condição quando tiveram filhos de baixo peso. Assim, ressalta-se a importância de conhecer e compreender os determinantes e condicionantes da saúde que estão associados ao processo de vulnerabilização e adoecimento feminino, para implementar estratégias de intervenção pertinentes no manejo da depressão, em todos os níveis de atenção à saúde.
\end{abstract}

Palavras-chave: Saúde da mulher; Paridade; Depressão; Brasil.

\begin{abstract}
Aim: Analyze the relationship between parity and depression in women of reproductive age interviewed in the National Health Survey, 2013. Methodology: Cross-sectional, population-based study carried out based on data from the Brazilian National Health Survey (PNS) - 2013. Included were records of women in reproductive period, aged between 18 and 49 years, totaling the sample with 15,344 women. Results: There was a statistically significant crude association between parity and depression (PR $=1.32$; 95\% CI: $1.20-1.46)$. There was also an association between having children with low weight and depression (PR $=1.64 ; 95 \% \mathrm{CI}: 1.24-2.18)$. Showing that women of older reproductive age and with three children or more are 36 times more likely to report a medical diagnosis of depression, and those who had children with low weight this probability almost doubled. Conclusion: Women of more advanced reproductive age, who had greater parity, were more likely to have depression, with a potential for this condition when they had low birth weight children. Thus, the importance of knowing and understanding the determinants and conditions of health that are associated with the process of vulnerability and female illness is emphasized, in order to implement relevant intervention strategies in the management of depression, at all levels of health care.
\end{abstract}


Keywords: Women's health; Parity; Depression; Brazil.

\section{Resumen}

Objetivo: Analizar la relación entre paridad y depresión en mujeres en edad reproductiva entrevistadas en la Investigación Nacional de Salud, 2013. Metodología: Estudio transversal, con enfoque poblacional, realizado a partir de datos de la Investigación Nacional de Salud de Brasil (2013). Se incluyeron registros de mujeres en período reproductivo, con edades comprendidas entre 18 y 49 años, totalizando la muestra con 15.344 mujeres. Resultados: Fue encontrada una asociación estadísticamente significativa entre la paridad y la depresión ( $\mathrm{RP}=1,32$; IC del 95\%: 1,20 - 1,46). También fue encontrada una asociación entre tener hijos con bajo peso y depresión ( $\mathrm{RP}=1,64$; IC del 95\%: 1,24 - 2,18). Estos resultados muestran que las mujeres en edad reproductiva mayor y con 3 hijos o más tienen 36 veces más probabilidades de reportar un diagnóstico médico de depresión y aquellas que tuvieron hijos con bajo peso esta probabilidad casi se duplicó. Conclusión: Las mujeres en edad reproductiva más avanzada y con mayor paridad tenían más probabilidades de tener depresión, con potencial para esta condición cuando tenían hijos con bajo peso al nacer. Así, se enfatiza la importancia de conocer y comprender los determinantes y condiciones de salud que se asocian al proceso de vulnerabilidad y enfermedad femenina, con el fin de implementar estrategias de intervención relevantes en el manejo de la depresión, en todos los niveles de la atención de salud.

Palabras clave: Salud de la mujer; Paridad; Depresión; Brasil.

\section{Introdução}

O histórico ginecológico e reprodutivo exerce influência sobre a saúde feminina (Câmara et al., 2015). Gestação e pós-parto têm sidos reconhecidos como fatores de risco para o aparecimento e piora de problemas na saúde mental (Bussel et al., 2006). Sugere-se que há frequência relevante de transtornos mentais em gestantes em acompanhamento pré-natal de baixo risco (Costa et al., 2018), e além disso, nos primeiros anos após o nascimento de um filho, a mulher apresentaria risco aumentado de apresentar um quadro de transtorno mental ou o recrudescimento de uma condição anteriormente existente (Brunette \& Dean, 2002).

Neste sentido, aspectos relacionados à paridade têm sido elencados como fatores relevantes para o surgimento da depressão (Clout \& Brown, 2015; Figueiredo e Conde, 2011; Schappin et al., 2013; Accortt et al., 2015). Mulheres são duas vezes mais acometidas por depressão, especialmente durante o período reprodutivo. Tal suscetibilidade decorre do aumento ou das oscilações nos níveis dos hormônios sexuais (Paykel, 2008; Molina et al., 2012; Stopa et al., 2015). Sendo observado maiores escores de depressão em mulheres multíparas, que tiveram gravidez não planejada e que apresentaram histórico de fetos natimorto (Golbasi et al., 2011). Embora haja evidência relatos de depressão correlacionados a primiparidade, parto prematuro, parto complicado, a experiência negativa com eventos de vida e preocupações sobre cuidados com os filhos (Evenson \& Simon, 2005; Woolhouse et al., 2015; Eutrope et al., 2014).

Além da ação hormonal e paridade, outros fatores estão fortemente associados ao processo de adoecimento por depressão em mulheres, tais como: desvantagens de gênero como violência sexual, baixa autonomia para tomar decisões, baixo suporte familiar (Patel et al., 2006; Vanwesenbeeck et al., 2014; Schetter et al., 2015), desigualdades socioeconômicas, baixo nível educacional, raça e aspectos culturais (Justo \& Calil, 2006; Bromet et al., 2011; Pabayo et al., 2014). Uma demonstração que elementos fisiológicos e sociais se relaciona à história reprodutiva e parecem contribuir para o aumento da carga de estresse a ser enfrentada ao longo da vida pela mulher (Velez et al., 2010). Ademais, estudos apontam a necessidade de maior investigação a respeito da saúde mental, principalmente por meio dos estudos populacionais, em virtude do pequeno número de investigações epidemiológicas de base populacional na área de saúde mental (Harpham et al., 2003; Maragno et al., 2006).

Desse modo, constitui-se como objetivo do estudo analisar a relação entre paridade e depressão em mulheres em idade reprodutiva entrevistadas na Pesquisa Nacional de Saúde, 2013.

\section{Metodologia}

Trata-se de um estudo transversal (Bordalo, 2006), de base populacional e com abordagem quantitativa realizado com 
base nos dados da Pesquisa Nacional de Saúde Brasileira (PNS) - 2013. A PNS produziu dados a respeito do estado de saúde e estilo de vida da população brasileira, bem como, da atenção a sua saúde, quanto ao acesso e uso dos serviços, às ações preventivas, à continuidade dos cuidados e ao financiamento da assistência (Damacena et al., 2014).

Responderam a PNS moradores em domicílios de todo o território nacional, excluídas áreas com características especiais e com pouca população, abrangendo 26 estados e o distrito federal. Ao todo foram realizadas entrevistas em 60.202 domicílios, totalizando 205.145 participantes, de ambos os sexos, com 18 anos ou mais de idade. Em cada domicílio, entrevistou-se toda a família para questões gerais sobre informações sociodemográficas e de saúde, e um morador selecionado respondeu questões adicionais sobre o estado de saúde.

Com vistas a responder ao objetivo do estudo se realizou recortes no banco de dados: inicialmente foram excluídos homens, totalizando 106.310 mulheres. Em seguida, excluiu-se as mulheres que mulheres com idade inferior a 18 anos e superior a 49 anos, ficando 52.069 mulheres. A pergunta proxy usada para este estudo foi: “Já ficou grávida na vida?". Por fim, das que responderam afirmativo ( $\mathrm{n}=16.811$ ), excluiu-se as mulheres que não tiveram filhos vivos, totalizando 15.344 mulheres, que compuseram amostra deste estudo. A obtenção da amostra estudada seguiu é apresentada em fluxograma na Figura 1.

A variável desfecho "ter depressão", foi avaliada por meio do autorrelato de diagnóstico de depressão, realizado por profissional especializado, conforme questionado pelos entrevistadores no momento de realização da PNS.

As variáveis independentes incluíram itens sociodemográficos, hábitos de vida, histórico reprodutivo e doenças crônicas, categorizadas da seguinte forma: a) A idade cronológica das mulheres foi dividida a partir da mediana, sendo dicotomizada em: 18 a 33 anos e 34 a 49 anos; b) A escolaridade foi categorizada em: escolarizado até ensino fundamental completo e ensino médio completo ou mais avançado; c) Para raça/cor, duas categorias foram criadas: branca, para indivíduos que se autodeclararam como brancas e negra, pardas e outras, para os indivíduos que se autodeclararam negras, pardas e outras; d) Para hábitos de vida, avaliou-se consumo de bebida alcoólica e prática de atividade física, nos últimos três meses.

A autopercepção do estado de saúde foi agrupada em saúde boa, para aqueles que escolheram as opções muito boa, boa e nem ruim/nem boa (escores 1, 2, e 3 na escala apresentada). Igualmente, determinou-se saúde precária para aqueles que avaliaram sua saúde como ruim ou muito ruim (escores 4 e 5 na escala apresentada).

Quanto às variáveis ginecológicas e histórico reprodutivo, foram incluídos os dados relativos à menarca, dicotomizada por meio da mediana, em: a) Menarca em idade igual ou inferior a 12 anos e em idade acima de 12 anos; b) Ter tido ou não relação sexual nos últimos 12 meses (sim ou não); c) Paridade, foi a dividida em até 3 filhos e 4 filhos ou mais; d) Tipo de parto em parto normal ou cesáreo; e) Filhos com baixo peso ao nascer e filhos pré-termo com peso adequado; f) idade da primeira gestação foi agrupada em menor que 19 anos e em 19 ou mais; g) ter realizado aborto provocado e aborto espontâneo (sim ou não).

As morbidades crônicas como hipertensão, diabetes, asma ou bronquite crônica, depressão, problema crônico na coluna, autorreferidas pelos informantes como presentes ou ausentes por diagnóstico médico foram agrupadas sim e não. 
Figura 1. Fluxograma do estudo
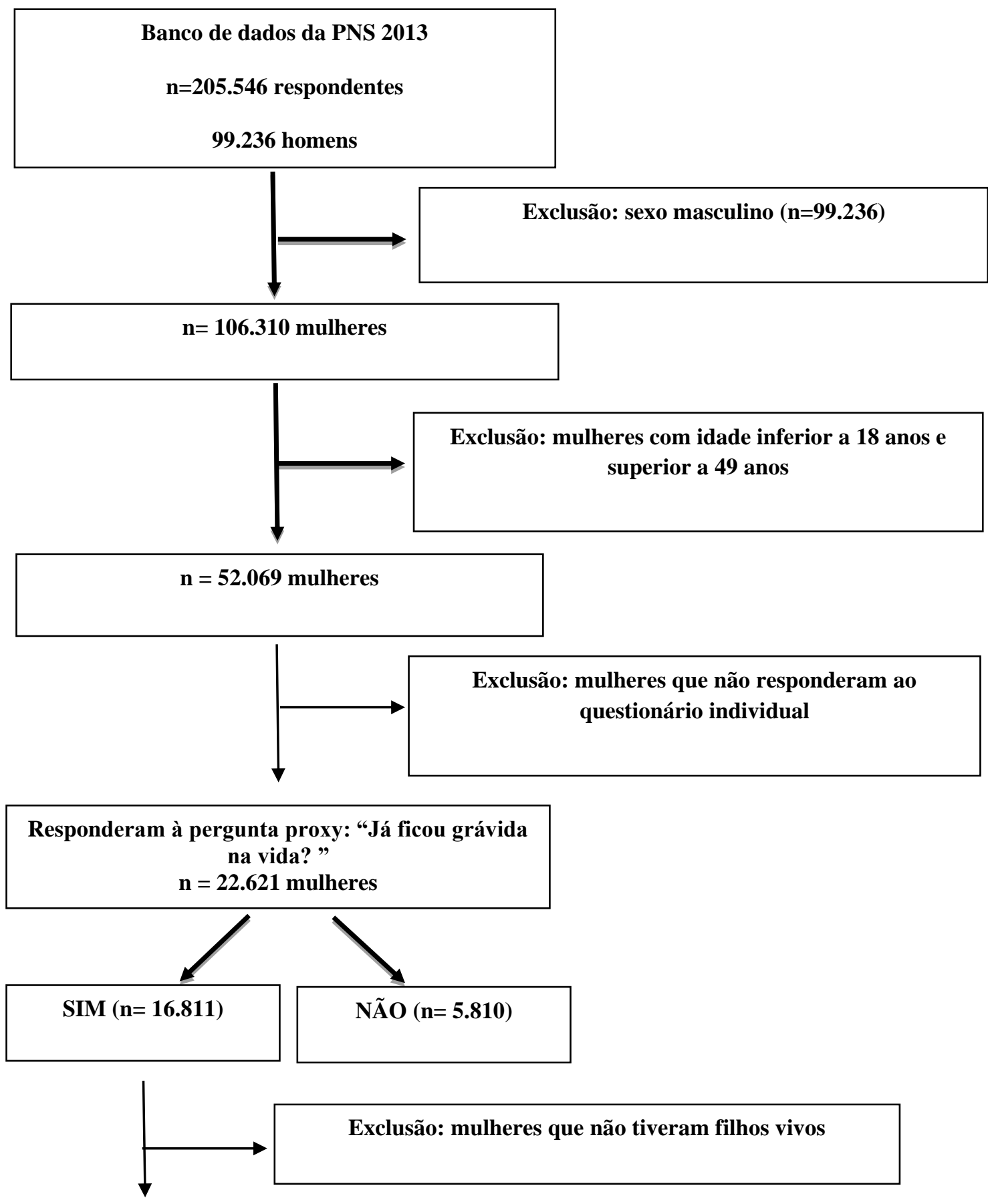

n= 15.344 mulheres

Fonte: Dados da Pesquisa (2021)

Para realização da análise estatística, foi utilizado o software STATA®, versão 9 (StatCorp, College Station, Texas, EUA). Foram analisadas as variáveis acima descritas como potenciais fatores de confundimento e de modificação de efeito da relação entre paridade e depressão.

Inicialmente, realizou-se uma análise descritiva onde foram estimadas frequências simples e relativas das variáveis independentes e dependente de acordo com as covariáveis do estudo. O teste de hipótese utilizado foi o qui-quadrado de Pearson e as variáveis foram descritas por meio de proporções. Foi utilizado paridade como estratificação fundamental em 
todas as análises. Em seguida, estimou-se a prevalência e razão de prevalência (RP) de depressão bruta e ajustada na amostra pelas covariáveis de interesse.

Para identificação dos potenciais modificadores de efeito, observou-se se as medidas de Odds Ratio (OR), por estrato, estavam inseridas no intervalo de confiança (IC 95\%) de cada covariável. Em seguida, o teste da razão da máxima verossimilhança foi conduzido para comparar os modelos brutos e ajustados, em ambos os grupos de paridade e confirmar a presença de variável de interação. Para a identificação de potenciais confundidores da associação de interesse, considerou-se a diferença relativa entre as medidas brutas e ajustadas maior ou igual a $15 \%$.

Para modelagem, inseriu-se no modelo inicial de cada grupo de paridade as covariáveis com $\mathrm{p} \leq 0,05$. Em seguida, foram construídos dois modelos múltiplos (por paridade) de regressão logística.

A regressão logística foi utilizada para estimar a razão de prevalência da associação entre paridade e depressão, estratificada por ter filhos com baixo peso ou não e ajustada pelos fatores que permaneceram significantes a 5\%. Em seguida, para cada modelo foi aplicado o teste de bondade do ajuste. Foi utilizado intervalo de confiança ao nível de 95\% (IC95\%) para inferência estatística. Foi realizado o teste de Hosmer-Lemeshow para avaliar o ajustamento do modelo final.

Referente aos aspectos éticos, a PNS foi aprovada pelo Conselho Nacional de Saúde - CNS e sua Comissão Nacional de Ética em Pesquisa - CONEP, sob o número 328.159, de 26 de junho de 2013. Todos os participantes assinaram o Termo de Consentimento Livre e Esclarecido, garantindo o sigilo dos dados.

\section{Resultados e Discussão}

A amostra do estudo foi constituída de 15.344 mulheres. A maioria da amostra foi composta por mulheres que estavam na faixa etária de 34 a 49 anos de idade (55.27\%; $\mathrm{n}=8.481$ ), que se autodeclararam de raça negra, parda e outras (65.88\%; $\mathrm{n}=10.108)$, com baixo grau de escolaridade (50.85\%; $\mathrm{n}=7.803$ ), não consumidoras regulares de bebidas alcoólicas $(83.72 \% ; \mathrm{n}=12.846)$ e que autorreferiram a própria saúde como boa $(95.37 \% ; \mathrm{n}=14.633)$.

Quanto às variáveis ginecológicas e reprodutivas, o perfil representativo do grupo apresentava menarca com mais de 12 anos de idade (56.4\%; $\mathrm{n}=8.649)$, presença de atividade sexual no último ano (86.5\%; $\mathrm{n}=13.276)$, primeira gestação antes dos 19 anos (69.34\%; $\mathrm{n}=10.639)$; ter realizado pelo menos um parto cesáreo $(53.0 \% ; \mathrm{n}=8.136)$; ter tido filhos pré-termo (84.8 $\% ; \mathrm{n}=13.006)$ e ter tido filhos com baixo peso para a idade gestacional $(85.8 \% ; \mathrm{n}=13.173)$. Sobre à presença de condições crônicas, os problemas de coluna foram os mais prevalentes (15.8\%) seguidos pela hipertensão (12.3\%) e depressão (9.1\%). Estes valores são mostrados na Tabela 1.

A partir dos resultados da primeira etapa da análise foi proposto um modelo para cada grupo. No modelo inicial foram inseridos os fatores cuja associação com depressão foi menor ou igual a $20 \%$ e permaneceram no modelo final aqueles com $\mathrm{p} \leq 0,05$ ou que perderam a significância estatística à medida que outras covariáveis foram retiradas. Os modelos finais foram ajustados por faixa etária e raça. 
Tabela 1. Características da população de estudo de acordo com a paridade.

\begin{tabular}{|c|c|c|c|}
\hline \multirow[b]{2}{*}{ Variáveis } & \multicolumn{2}{|c|}{ Paridade } & \multirow[b]{2}{*}{ Total n (\%) } \\
\hline & Até 3 filhos n (\%) & $\begin{array}{c}4 \text { filhos ou mais } \\
\text { n }(\%)\end{array}$ & \\
\hline \multicolumn{4}{|l|}{ Faixa Etária } \\
\hline 18 a 33 anos & $5.213(75.96)$ & $1.650(24.04)$ & $6.863(44.73)$ \\
\hline 34 a 49 & $4.952(58.39)$ & $3.529(41.61)$ & $8.481(55.27)$ \\
\hline \multicolumn{4}{|l|}{ Raça } \\
\hline Branca & $3.915(74.77)$ & $1.321(25.23)$ & $5.236(34.12)$ \\
\hline Negra, Parda e outras & $6.250(61.83)$ & $3.858(38.17)$ & $10.108(65.88)$ \\
\hline \multicolumn{4}{|l|}{ Escolaridade } \\
\hline $\begin{array}{l}\text { Até Ensino fundamental } \\
\text { completo }\end{array}$ & $4.135(52.99)$ & $3.668(47.01)$ & $7.803(50.85)$ \\
\hline $\begin{array}{l}\text { Ensino Médio completo ou } \\
\text { mais }\end{array}$ & $6.030(79.96)$ & $1.511(20.04)$ & $7.541(49.15)$ \\
\hline \multicolumn{4}{|l|}{ Consumo Bebida } \\
\hline Não & $8.410(65.47)$ & $4.436(34.53)$ & $12.846(83.72)$ \\
\hline Sim & $1.755(70.26)$ & $743(29.74)$ & $2.498(6.28)$ \\
\hline \multicolumn{4}{|l|}{ Saúde autorreferida } \\
\hline Boa & $9.837(67.22)$ & $4.796(32.78)$ & $14.633(95.37)$ \\
\hline Precária & $328(46.13)$ & $383(53.87)$ & $711(4.63)$ \\
\hline \multicolumn{4}{|l|}{ Idade da Menarca } \\
\hline$>12$ anos & $5.699(65.89)$ & $2.950(34.11)$ & $8.649(56.37)$ \\
\hline$\leq 12$ anos & $4.466(66.71)$ & $2.229(33.29)$ & $6.695(43.63)$ \\
\hline \multicolumn{4}{|l|}{ Relação Sexual*** } \\
\hline Não & $1.231(59.53)$ & $837(40.47)$ & $2.068(13.48)$ \\
\hline Sim & $8.934(67.29)$ & $4.342(32.71)$ & $13.276(86.52)$ \\
\hline \multicolumn{4}{|l|}{ Idade da Primeira Gestação } \\
\hline Menor que 19 anos & $7.964(74.86)$ & $2.675(25.14)$ & $10.639(69.34)$ \\
\hline 19 ou mais & $2.201(46.78)$ & $2.504(53.22)$ & $4.705(30.66)$ \\
\hline \multicolumn{4}{|l|}{ Tipo de Parto } \\
\hline Normal & $4.487(62.25)$ & $2.721(37.75)$ & $7.208(46.98)$ \\
\hline Cesáreo & $5.678(69.79)$ & $2.458(30.21)$ & $8.136(53.02)$ \\
\hline \multicolumn{4}{|l|}{ Filhos Baixo Peso } \\
\hline Não & $9.081(68.94)$ & $4.092(31.06)$ & $13.173(85.85)$ \\
\hline Sim & $1.084(49.93)$ & $1.087(50.07)$ & $2.171(14.15)$ \\
\hline \multicolumn{4}{|l|}{ Filhos Prematuro } \\
\hline Não & $8.809(67.73)$ & $4.197(32.27)$ & $13.006(84.76)$ \\
\hline Sim & $1.356(58.00)$ & $982(42.00)$ & $2.338(15.24)$ \\
\hline \multicolumn{4}{|l|}{ Aborto Espontâneo } \\
\hline Não & $8.248(69.65)$ & $3.594(30.35)$ & $11.842(77.18)$ \\
\hline Sim & $1.917(54.74)$ & $1.585(45.26)$ & $3.502(22.82)$ \\
\hline \multicolumn{4}{|l|}{ Aborto Provocado } \\
\hline Não & $9.878(66.64)$ & $4.945(33.36)$ & $14.823(96.60)$ \\
\hline Sim & 287(55.09) & 234(44.91) & $521(3.40)$ \\
\hline \multicolumn{4}{|l|}{ Hipertensão } \\
\hline Não & $9.148(68.01)$ & 4.302(31.99) & $13.450(87.66)$ \\
\hline Sim & $1.017(53.70)$ & $877(46.30)$ & $1.894(12.34)$ \\
\hline \multicolumn{4}{|l|}{ Depressão } \\
\hline Não & $9.334(66.90)$ & $4.618(33.10)$ & $13.952(90.93)$ \\
\hline
\end{tabular}




\begin{tabular}{lccc} 
Sim & $831(59.70)$ & $561(40.30)$ & $1.392(9.07)$ \\
Diabetes & & & \\
Não & $9.962(66.55)$ & $5.008(33.45)$ & $14.970(97.56)$ \\
Sim & $203(54.28)$ & $171(45.72)$ & $374(2.44)$ \\
Asma ou Bronquite crônica & & & \\
Não & $9.671(66.27)$ & $4.923(33.73)$ & $14.594(95.11)$ \\
Sim & $494(65.87)$ & $256(34.13)$ & $750(4.89)$ \\
Problema crônico na coluna & & & \\
Não & $8.692(67.33)$ & $4.217(32.67)$ & $12.909(84.13)$ \\
Sim & $1.473(60.49)$ & $962(39.51)$ & $2.435(15.87)$ \\
\hline
\end{tabular}

Fonte: Banco de dados da PNS, MS/IBGE (2013)

Observou-se associação bruta estatisticamente significante entre a paridade e depressão (RP= 1.32; IC95\%: 1.20 1.46). Na análise bivariada, observou-se que as medidas de ponto estrato específico estavam contidas no intervalo de confiança correlato em todas as covariáveis estudadas. Esses resultados encontram-se evidenciados na Tabela 2. Além disso, o teste da razão da máxima verossimilhança conduzido para comparar os modelos brutos e saturados, em ambos os grupos, não evidenciou diferença significativa, indicado que não houve covariável modificadora de efeito da associação em estudo.

Tabela 2. Razões de prevalência brutas e ajustadas de acordo com as covariáveis analisadas, entre paridade e depressão e os respectivos IC $95 \%$.

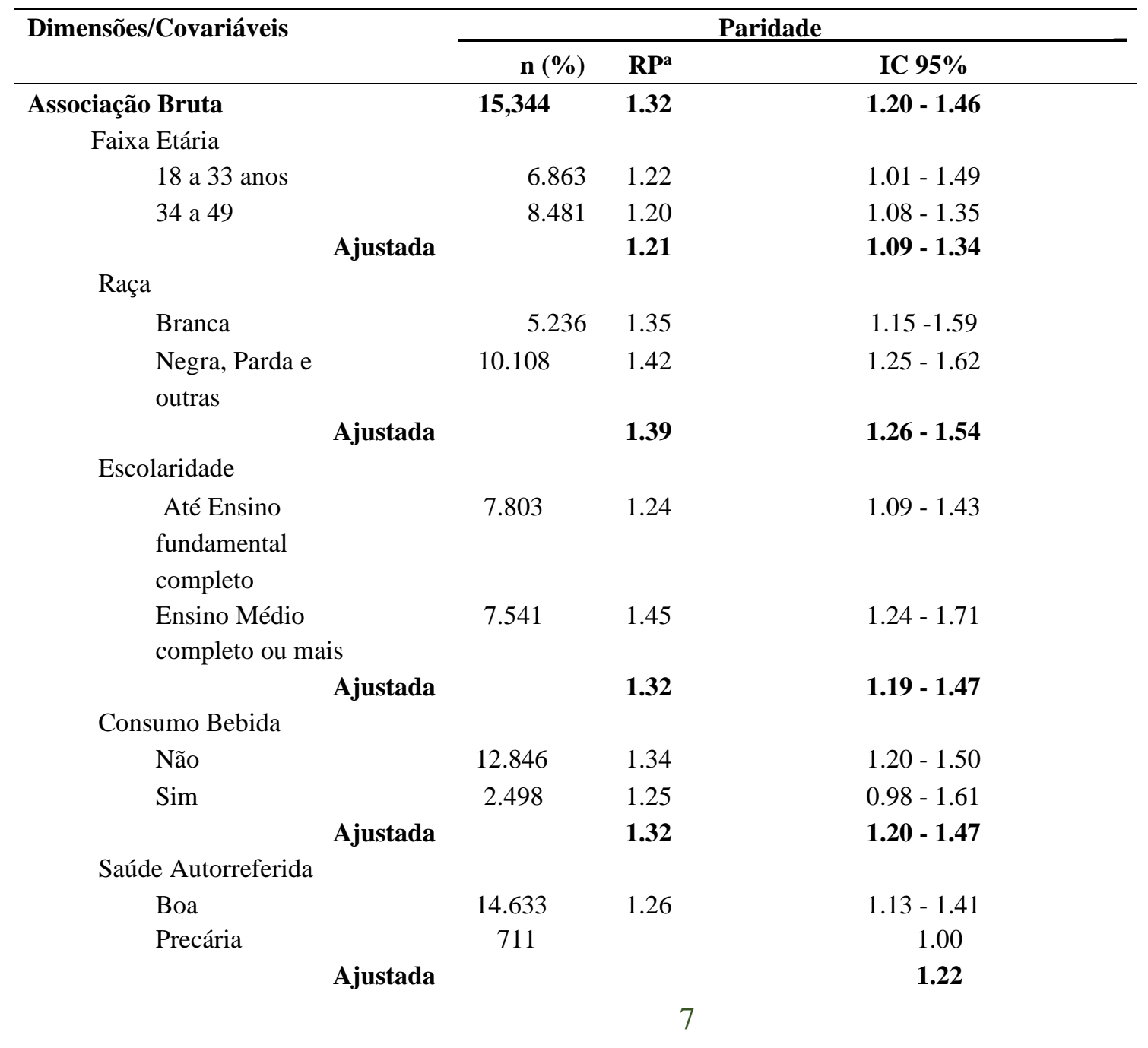


Idade da Menarca

$$
\begin{aligned}
& >12 \text { anos } \\
& \leq 12 \text { anos }
\end{aligned}
$$

Relação Sexual**

Não

Ajustada
1.29

1.36

1.32

1.28

1.31

1.30

Idade da Primeiro Gestação 19 ou mais menor que 19 anos 10.639

1.34

4.705

Ajustada

Tipo de Parto

Normal

Cesário

Ajustada

Ter filhos Baixo Peso
Não

Sim

Ajustada

13.173

2,171

1.22

1.66

1.29

Ter Filhos Pré- termo

$$
\begin{aligned}
& \text { Não } \\
& \text { Sim }
\end{aligned}
$$

13,006

1.28

2.338

1.27

1.28

Aborto Espontâneo

Não
Sim

Aborto Provocado

Não

Sim

Hipertensão
Não
Sim

Diabetes
Não
Sim

Asma ou Bronquite crônica

$$
\text { Ajustada }
$$

11.842

1.28

3.502

1.23

1.26

$$
14.823
$$

1.31

1.48

1.31

Ajustada

$$
521
$$

$13.450 \quad 1.30$

$1.894 \quad 1.05$

Ajustada

1.23

14.970

1.33

0.96

$\mathbf{1 . 3 1}$

14.594

1.29

Problema crônico na coluna
1.60

Ajustada

Não
Sim

Não

750

$\mathbf{1 . 3 2}$

12.909

2.435

Ajustada
1.35

1.08

$\mathbf{1 . 2 6}$
$1.13-1.49$

$1.17-1.58$

1.20 - 1.47

$1.01-1.62$

$1.17-1.47$

1.18 - 1.45

$1.18-1.52$

$1.17-1.71$

1.22 - 1.52

$1.14-1.56$

$1.20-1.57$

$1.22-1.50$

$1.09-1.37$

$1.30-2.12$

1.17 - 1.43

$1.15-1.45$

$1.03-1.56$

1.16 - 1.42

$1.13-1.46$

$1.03-1.48$

1.15 - 1.41

$1.18-1.45$

$0.93-2.34$

1.19 - 1.46

$1.15-1.46$

$0.87-1.27$

1.11 - 1.36

$1.20-1.48$

$0.59-1.55$

1.19 - 1.45

$1.16-1.44$

$1.20-2.12$

1.20 - 1.46

$1.19-1.54$

0.92 - 1.29

1.13 1.39

Fonte: Banco de dados da PNS, MS/IBGE (2013). 
Referente ao modelo final para os grupos de paridade ajustados por faixa etária e raça mostrado na Tabela 3. O teste de bondade do ajustamento apontou a adequação do modelo final da análise de regressão de logística para associação entre ter filhos com baixo peso e depressão, mostrando que as mulheres com 3 filhos ou mais apresentam 36 vezes mais chances de referir diagnóstico médico de depressão, e aquelas que tiveram filho com baixo peso essa probalidade foi quase duplicada.

Tabela 3 - Análise estratificada e regressão logística (modelo final) para a associação entre paridade e depressão por filhos com baixo peso de nascimento dos filhos segundo covariáveis

\begin{tabular}{|c|c|c|c|c|}
\hline \multirow[b]{2}{*}{ Exposição } & \multicolumn{2}{|c|}{ Ter filho sem baixo peso } & \multicolumn{2}{|c|}{ Ter filho com baixo peso } \\
\hline & $\begin{array}{l}\text { RP' }^{\mathrm{I}} \text { Bruta } \\
\text { (IC95\%) }\end{array}$ & 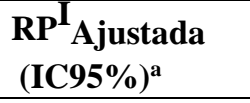 & $\begin{array}{l}\text { RP'Bruta } \\
\text { (IC95\%) }\end{array}$ & $\begin{array}{l}\text { RP'Ajustada } \\
\text { (IC95\%) }^{a}\end{array}$ \\
\hline \multicolumn{5}{|l|}{ Paridade } \\
\hline Até 3 filhos & 1,00 & 1,00 & 1,00 & 1,00 \\
\hline $\begin{array}{l}4 \text { filhos ou } \\
\text { mais }\end{array}$ & $\begin{array}{l}1.36(1.22- \\
1.53)\end{array}$ & $\begin{array}{c}1.20(1.06- \\
1.37)\end{array}$ & $\begin{array}{c}1.36(1.22- \\
1.53)\end{array}$ & $\begin{array}{c}1.64(1.24- \\
2.18)\end{array}$ \\
\hline
\end{tabular}

i Razão de prevalência

aAjustado por faixa etária e raça

Ter filho sem baixo peso: Teste de Hosmer-Lemeshow = 0.92;

Ter filho com baixo peso: Teste de Hosmer-Lemeshow $=0.40$

Fonte: Autores.

O presente estudo monstrou que mulheres em idade reprodutiva mais avançada e que tiveram maior paridade, apresentaram mais chances de ter depressão, demonstrando que ter maior número de filhos é um importante fator preditor de depressão. Ressalta-se ainda, que se o filho tiver baixo peso, essa condição é potencializada, uma vez que observou-se no decorrer dos resultados que o ajuste por idade e raça, reduzindo a medida de ponto de 1,36 para 1,20 as chances da mulher ter depressão, já a presença de filho de baixo peso aumenta a medida de ponto para 1,64, mesmo que ajustando o efeito da idade e da raça. Dessa forma, apontando que a saúde mental é efetivamente afetada pela paridade (ou múltipla maternidade) e crianças que demandam mais cuidados (baixo peso) parecem sobrepor esse efeito.

Em concordância com os achados do presente estudo, pesquisa realizada sobre os efeitos pscossciais da gestação e maternidade obteve que a relação entre maior paridade e depressão pode se dever ao estresse, sobrecarga do cuidado com os outros filhos e a falta de apoio social (Hung, 2007). Ademais, também são relatados fatores como a relação entre maior número de filho e a falta de recursos financeiros, dificuldades em encontrar trabalho, vagas em creches, outro aspecto elencado é o número de filhos vivos está relacionada com a paternidade, pois muitas mães multíparas relataram ter filhos de companheiros anteriores e ter medo que os atuais maridos ou companheiros passassem a maltratar os filhos mais velhos delas dos quais não eram pais biológicos (Viegas et al., 2008). Sendo crescido aos aspectos já mencionados, multíparas relataram níveis mais baixos de apoio social e satisfação conjugal (Sockol \& Battle, 2015). Se contrapondo ao exposto ao presente estudo realizado na Hungria (Castordai et al., 2007) ressalta que na depressão pós-parto o maior número de filhos representa uma probabilidade significativamente menor de vulnerabilidade no caso de mulheres multíparas para esse transtorno, enfatizando que risco de depressão pós-parto pode ser modificado pelos dados da história sociodemográfica e obstétrica.

No que se refere a idade, estudos nacionais e internacionais indicam que mulheres mais novas estão mais vulneráveis a depressão (Hung, 2007). Entrentanto, o presente estudo encontrou que mulheres em idade reprodutiva mais avançada tiveram mais chances deter depressão, corroborando os achados de pesquisas internacionais de Stordal et al. (2003) e Aasheim et al. (2012). Destaca-se como possível explicação para essa associação a grande variabilidade hormonal, a exemplo dos períodos pré menstruais e puerperal, vivenciado na perimenopausa (Altemus, 2006; Douma et al., 2005). Estudos mais atuais sugerem 
evidências preliminares de uma relação curvilínea entre a idade da mãe no primeiro parto e a depressão, sugerindo que a maternidade, tanto em mulheres jovens (20 anos ou menos) quanto em idades mais avançadas (após os 30 anos de idade) afeta negativamente a saúde mental das mulheres e que esses efeitos persistem ao longo da vida (Koropeckyj-Cox et al., 2007).

Outro aspecto evidenciado na presente pesquisa foi que, mulheres que se auto declararam negras, pardas e outras, que possuíam mais filhos, apresentaram mais chances de ter depressão. Uma revisão sistemática sobre raça/cor da pele e saúde mental no contexto brasileiro, contendo cinco estudos com mulheres grávidas ou que tiveram filhos nascidos vivos recentemente, mostrou que análises multivariadas, com significância estatística apontaram associações positivas entre a raça/cor não branca e transtornos mentais (Smolen \& Araújo, 2017), corroborando os achados do presente estudo. Entretanto, em outros países, mulheres brancas apresentam maior prevalência de depressão (Riolo et al., 2005; Watson et al., 2012).

Um fator potencializador para depressão, encontrado no presente estudo, foi o relato de mulheres que tiveram filhos com baixo peso ao nascer. Estudos relatam que reações psicológicas das mães, como depressão e ansiedade, se correlacionam fortemente com a presença de um trauma inicial relacionada ao nascimento, relacionado ao peso do bebê (Schappin et al., 2013; Eutrope et al., 2014). Nesse sentido, mães de bebês prematuros podem ter maior risco de depressão no período pós-natal do que mães de bebês a termo, e mães de lactentes de baixo peso possuem risco contínuo de depressão, durante primeiro ano de vida do bebê (Vigod et al., 2010).

Estudo de revisão sistemática e meta-análise (Jarde et al., 2016), evidenciou que depressão pré-natal não tratada foi associada com criança de baixo peso e com a menor idade gestacional. Outros autores enfatizaram que o efeito da depressão durante a gestação é mais relacionado ao baixo peso do bebê ao nascer do que sobre a idade gestacional (Schappin et al., 2013). Hipóteses a respeito da influência do uso de antidepressivos durante a gravidez está associada a efeitos no crescimento fetal vêm sendo debatidas, a saber: o uso de medicamentos pode reduzir os sintomas depressivos e, assim, reduzir o impacto da depressão sobre o crescimento fetal, ou, de forma interativa, os antidepressivos podem potencializar os efeitos da depressão no desenvolvimento fetal. Embora nesse estudo não tenha sido analisado o uso de medicamentos antidepressivos durante a gestação, chama-se a atenção para essa discussão pela importância clínica de prescrições seguras durante a gestação (Lewis et al., 2016).

\section{Considerações Finais}

Os achados decorrentes deste estudo ressaltam a importância de conhecer e compreender os determinantes e condicionantes da saúde que estão associados ao processo de vulnerabilização e adoecimento feminino, no sentido de implementar estratégias de intervenção que sejam pertinentes com o manejo da depressão, em todos os níveis de atenção à saúde. A depressão é um transtorno que acomete mais as mulheres, o que tem sido amplamente confirmado pelos estudos epidemiológicos. Através dos achados do presente estudo, observou-se mulheres negras em idade reprodutiva mais velhas, que tiveram maior paridade, tiveram mais chances de ter depressão.

Uma limitação deste estudo decorre de sua natureza transversal e por ter sido realizado por meio do autorrelato dificultando a realização de inferências de causalidade. Contudo, os resultados aqui expostos ampliam a compreensão do adoecimento feminino para além dos fatores puramente biológicos, tendo em vista que demonstra a importância dos determinantes sociais no processo de adoecimento das mulheres em idade reprodutiva.

Com esse estudo, espera-se fomentar o debate do tema, colaborar na construção de políticas públicas que ajudem a intervir precocemente na depressão durante o período gestacional. Sugere-se a elaboração de novas pesquisas principalmente quanto a estratégias de enfrentamento e não cronificação de modo a reduzir os impactos negativos desse fenômeno. 


\section{Referências}

Aasheim, V., Waldenström, U., Hjelmstedt, A., Rasmussen, S., Pettersson, H., \& Schytt, E. (2012) Associations between advanced maternal age and psychological distress in primiparous women, from early pregnancy to 18 months postpartum. BJOG: An International Journal of Obstetrics \& Gynaecology, 119(9), 1108-1116.

Accortt, E. E., Cheadle, A. C., \& Schetter, C. D. (2015) Prenatal depression and adverse birth outcomes: an updated systematic review. Maternal and child health journal, 19(6), 1306-1337.

Altemus, M. (2006) Sex differences in depression and anxiety disorders: potential biological determinants. Hormones and behavior, 50(4), 534-538.

Bordalo, A. A. (2006) Estudo transversal e/ou longitudinal. Revista Paraense de Medicina Vol. 20(4), 1.

Bromet, E., Andrade, L. H., Hwang, I., Sampson, N. A., Alonso, J., De Girolamo, G., \& Kessler, R. C. (2011) Cross-national epidemiology of DSM-IV major depressive episode. BMC medicine, 9(1), 1-16.

Brunette, M. F., Dean W. (2002) Community mental health care for women with severe mental illness who are parents. Community Mental Health Journal, Heidelberg-Germany, 38 (2), 153-165.

Câmara, S. M. A., Pirkle, C., Moreira, M. A., Vieira, M. C. A., Vafaei, A., \& Maciel, Á. C. C. (2015) Early maternal age and multiparity are associated to poor physical performance in middle-aged women from Northeast Brazil: a cross-sectional community based study. BMC women's health, 15(1), 1-10.

Clout, D., \& Brown, R. (2015) Sociodemographic, pregnancy, obstetric, and postnatal predictors of postpartum stress, anxiety and depression in new mothers. Journal of Affective Disorders, 188, 60-67.

Costa, D. O., Souza, F. I. S. D., Pedroso, G. C., \& Strufaldi, M. W. L. (2018) Mental disorders in pregnancy and newborn conditions: longitudinal study with pregnant women attended in primary care. Ciencia \& saude coletiva, 23, 691-700.

Csatordai, S., Kozinszky, Z., Devosa, I., Tóth, É., Krajcsi, A., Sefcsik, T., \& Pál, A. (2007) Obstetric and sociodemographic risk of vulnerability to postnatal depression. Patient Education and Counseling, 67(1-2), 84-92.

Damacena, G. N., Szwarcwald, C. L., Malta, D. C., Souza Júnior, P. R. B. D., Vieira, M. L. F. P., Pereira, C. A., \& Silva Júnior, J. B. D. (2015) The development of the National Health survey in Brazil, 2013. Epidemiologia e Serviços de Saúde, 24, 197-206.

Douma, S. L., Husband, C., O'donnell, M. E., Barwin, B. N., \& Woodend, A. K. (2005) Estrogen-related mood disorders: reproductive life cycle factors. Advances in Nursing Science, 28(4), 364-375.

Eutrope, J., Thierry, A., Lempp, F., Aupetit, L., Saad, S., Dodane, C., \& Rolland, A. C. (2014) Emotional reactions of mothers facing premature births: study of 100 mother-infant dyads 32 gestational weeks. PloS one, 9(8), e104093.

Evenson, R. J., \& Simon, R. W. (2005) Clarifying the relationship between parenthood and depression. Journal of health and Social Behavior, 46(4), 341-358.

Figueiredo, B., \& Conde, A. (2011) Anxiety and depression symptoms in women and men from early pregnancy to 3-months postpartum: parity differences and effects. Journal of affective disorders, 132(1-2), 146-157.

Golbasi, Z., Kelleci, M., Kisacik, G., \& Cetin, A. (2010) Prevalence and correlates of depression in pregnancy among Turkish women. Maternal and child health journal, 14(4), 485-491.

Harpham, T., Reichenheim, M., Oser, R., Thomas, E., Hamid, N., Jaswal, S., \& Aidoo, M. (2003) Measuring mental health in a cost-effective manner. Health policy and planning, 18(3), 344-349.

Hung, C. H. (2007) The psychosocial consequences for primiparas and multiparas. The Kaohsiung journal of medical sciences, 23(7), 352-360.

Jarde, A., Morais, M., Kingston, D., Giallo, R., MacQueen, G. M., Giglia, L., \& McDonald, S. D. (2016) Neonatal outcomes in women with untreated antenatal depression compared with women without depression: a systematic review and meta-analysis. JAMA psychiatry, $73(8), 826-837$.

Justo, L. P., \& Calil, H. M. (2006) Depression: does it affect equally men and women? Archives of Clinical Psychiatry (São Paulo), 33(2), 74-79.

Koropeckyj-Cox, T., Pienta, A. M., \& Brown, T. H. (2007). Women of the 1950s and the "normative" life course: The implications of childlessness, fertility timing, and marital status for psychological well-being in late midlife. The International Journal of Aging and Human Development, 64(4), 299-330.

Lewis, A. J., Austin, E., \& Galbally, M. (2016) Prenatal maternal mental health and fetal growth restriction: a systematic review. Journal of developmental origins of health and disease, 7(4), 416-428.

Maragno, L., Goldbaum, M., Gianini, R. J., Novaes, H. M. D., \& César, C. L. G. (2006) Prevalence of common mental disorders in a population covered by the Family Health Program (QUALIS) in São Paulo, Brazil. Cadernos de saude publica, 22(8), 1639-1648.

Molina, M. R. A. L., Wiener, C. D., Branco, J. C., Jansen, K., Souza, L. D. M. D., Tomasi, E., \& Pinheiro, R. T. (2012) Prevalence of depression in users of primary care settings. Archives of Clinical Psychiatry (São Paulo), 39(6), 194-197.

Pabayo, R., Kawachi, I., \& Gilman, S. E. (2014) Income inequality among American states and the incidence of major depres sion. J Epidemiol Community Health, 68(2), 110-115.

Patel, V., Kirkwood, B. R., Pednekar, S., Weiss, H., \& Mabey, D. (2006) Risk factors for common mental disorders in women: Population-based longitudinal study. The British Journal of Psychiatry, 189(6), 547-555. 
Research, Society and Development, v. 10, n. 4, e59910414516, 2021

(CC BY 4.0) | ISSN 2525-3409 | DOI: http://dx.doi.org/10.33448/rsd-v10i4.14516

Paykel, E. S. (2008) Basic concepts of depression. Dialogues in Clinical Neuroscience 10 (3), 279-289.

Riolo, S. A., Nguyen, T. A., Greden, J. F., \& King, C. A. (2005) Prevalence of depression by race/ethnicity: findings from the National Health and Nutrition Examination Survey III. American journal of public health, 95(6), 998-1000.

Schappin, R., Wijnroks, L., Venema, M. M. U., \& Jongmans, M. J. (2013) Rethinking stress in parents of preterm infants: a meta-analysis. PloS one, 8(2), e54992.

Schetter, C. D., \& Tanner, L. (2012) Anxiety, depression and stress in pregnancy: implications for mothers, children, research, and practice. Current opinion in psychiatry, 25(2), 141.

Smolen, J. R. \& Araújo, E. M. (2017) Raça/cor da pele e transtornos mentais no Brasil: uma revisão sistemática. Ciência \& Saúde Coletiva, 22(12), 40214030 .

Sockol, L. E., \& Battle, C. L. (2015) Maternal attitudes, depression, and anxiety in pregnant and postpartum multiparous women. Archives of women's mental health, 18(4), 585-593.

Stoppa, S. R., Malta, D. C., Oliveira, M. M., Lopes, C. S., Menezes, P. R., \& Kinoshita, R. T. (2015) Prevalence of self-reported depression in Brazil: 2013 National Health Survey results. Rev Bras Epidemiol, 170-80.

Stordal, E., Mykletun, A., \& Dahl, A. A. (2003) The association between age and depression in the general population: a multivariate examination. Acta psychiatrica scandinavica, 107(2), 132-141.

Van Bussel, J. C., Spitz, B., \& Demyttenaere, K. (2006) Women’s Mental Health Before, During, and After Pregnancy: A Population-Based Controlled Cohort Study. Birth, 33(4), 297-302.

Vanwesenbeeck, I., Ten Have, M., \& de Graaf, R. (2014) Associations between common mental disorders and sexual dissatisfaction in the general population. The British Journal of Psychiatry, 205(2), 151-157.

Velez, M. P., Alvarado, B. E., Rosendaal, N., da Câmara, S. M., Belanger, E., Richardson, H., \& Pirkle, C. M. (2019) Age at natural menopause and physical functioning in postmenopausal women: the Canadian Longitudinal Study on Aging. Menopause, 26(9), 958.

Viegas, L. M., Silva, G. A., Cecchini, M., Felipe, R., Otta, E., Bussab, V. S. R. (2008) Depressão pós-parto, histórico reprodutivo materno e apoio social. Boletim do Inst Saúde, 9-11.

Vigod, S. N., Villegas, L., Dennis, C. L., \& Ross, L. E. (2010) Prevalence and risk factors for postpartum depression among women with preterm and low-birth-weight infants: a systematic review. BJOG: An International Journal of Obstetrics \& Gynaecology, 117(5), 540-550.

Watson, K. T., Roberts, N. M., \& Saunders, M. R. (2012) Factors associated with anxiety and depression among African American and White women. International Scholarly Research Notices, 2012.

Woolhouse, H., Gartland, D., Mensah, F., \& Brown, S. J. (2015) Maternal depression from early pregnancy to 4 years postpartum in a prospective pregnancy cohort study: implications for primary health care. BJOG: An International Journal of Obstetrics \& Gynaecology, 122(3), 312-321. 